\title{
A Study of the Solid Freeform Fabrication (SFF) System with Dual Laser System*
}

\author{
Dong Soo KIM**, Young Jin AHN**, Won Hee LEE**, \\ Sung Woo BAE** and Kyung Hyun $\mathrm{CHOI}^{* * *}$
}

\begin{abstract}
A solid freeform fabrication (SFF) system using selective laser sintering (SLS) is currently recognized as a leading process and SLS extends the applications to machinery and automobiles due to the various materials employed. In order to develop a more elaborate and rapid system for fabricating large objects compared to existing SLS, this study employs a new selective dual-laser sintering (SDLS) process. It contains a 3-axis, dynamic focusing scanner system for scanning large areas instead of the existing $f \theta$ lens used in commercial SLS. Therefore, the unique scanning path generation is necessary to eliminate the factors of quality deterioration in case of fabricating larger objects. Also, this paper will address development of an SFF system which employs the dual laser system and the unique scanning device. Experiments were performed to evaluate the effect of a scanning path and fabrication parameters on sintering process and to fabricate the various $3 \mathrm{D}$ objects using polymer powder.
\end{abstract}

Key Words: SFF (Solid Freeform Fabrication), Optical 3D Scanner, SLS (Selective Laser Sintering), Optimal Sintering Parameters, Scanning Path

\section{Introduction}

Since the life cycle of a product is decreasing due to rapid industrial development and the diverse needs of customers, the reduction of the time required for new product development is a significant issue. A solid freeform fabrication (SFF) system, known as Rapid prototyping (RP) technology, used since the late 1980s, has taken its place in CAD/CAM and has been expected to cope with the dynamic manufacturing environment. RP is a material additive process, in which a 3-D computer model is sliced and reassembled in real space, layer-by-layer ${ }^{(1)}$. Based on the original form of the material used and the hardening method, the various RP systems, such as SLA (stereo lithography apparatus), SLS (selective laser sintering), LOM (laminated object manufacturing), FDM (fused deposit modeling), and SGC (solid ground curing), were in-

* Received 22nd March, 2006 (No. 06-5045)

** Intelligence and Precision Machinery Research Division/IT Machinery Research Center, Korea Institute of Machinery \& Materials, 171 Jang-Dong, Yuseong-Gu, Daejeon 305-343, Korea. E-mail: kds671@kimm.re.kr

*** School of Mechanical Engineering, Cheju National University, 66 Jejudaehakno, Jejusi, Jeju Special SelfGoverning Province 690-756, Korea troduced to the market ${ }^{(2)}$. The SLS process known as solid freeform fabrication (SFF), creates 3-D objects, layer-bylayer, using powdered materials with heat generated by a $\mathrm{CO}_{2}$ laser. Using the SLS process, prototypes were produced with various uses of thermoplastic, metal composite and ceramic composite powder ${ }^{(3)-(6)}$.

Compared with the existing material removal process, SFF system has the multiple capabilities of producing prototypes rapidly, forming complex internal shapes, easily performing the modification process, and fabricating multi-products. On the contrary, one of the shortcomings of SFF is that the surface quality of prototypes and the processing time are affected significantly by the fabricating configuration. To develop SFF system being capable of large size fabrication $(500 \times 800 \times 600 \mathrm{~mm}, \mathrm{~W} \times \mathrm{D} \times \mathrm{H})$, the dual laser system should be employed, which can scan divided two regions individually. The laser scanner highly affects the precision and efficiency of SLS machine. The laser scanner is in cooperation with both the generation of scan paths from a sliced section and the scan control to follow the generated paths.

To generate scan paths fast, adaptive paths according to the geometrical shape of each layer are needed ${ }^{(8)-(10)}$. Chen ${ }^{(7)}$ developed an intelligent scan method to reduce curling, shrinkage, and growth of the fabricated part. Also 
Bonus-Z model was developed to compensate bottom growth at the several bottom surfaces. Park ${ }^{(11)}$ enhanced the quality of fabricated part by generating scan path from the directly sliced section from STL file. Yang et al. ${ }^{(12)}$ developed curling and shrinkage phenomena more by relieving thermal stresses differences between neighboring regions in the same layer. Yang et al. ${ }^{(13)}$ studied effective scan method by generating scan path with offsets from contour.

Also, several build parameters such as laser power, heat temperature, and powder cartridge feed rate should be selected carefully. Above all, it takes longer to prepare a fabrication, such as supplying powder and moving the platform ad compared to the sintering process. Therefore, determination of the fabricating parameters is considered to be the most important process condition in the RP operation $^{(14),(15)}$.

This research addresses development of a SFF system which contains dual lasers and a 3-axis dynamic focusing scanner system to enable large-area real object duplication. To evaluate the applicability of the SFF system developed, experiments were conducted with optimal fabricating parameters.

\section{Development of SFF System Using Dual Laser}

As illustrated in Fig. 1, an SFF system developed is composed of a laminating module that supplies and transfers the powder, a heating module to preheat the powder, a nitrogen supply module to create a nitrogen atmosphere, the dual laser module that supplies the laser to a large area, laser units, and a control module to control the entire system.

\subsection{Laminating module}

The SLS process used CAD data or 3D images to fabricate a $3 \mathrm{D}$ structure. The powder was deposited layer by layer and a structure was constructed through laser sin- tering. For this reason, controls in the $z$ axis to deposit powder and powder transfer in the $x$ axis using a roller are closely related to the precision of a $3 \mathrm{D}$ structure. As illustrated in Fig. 1, the laminating module consisted of a build room, 2 feeding rooms, a LM guide, and servo and stepping motors. Figure 2 (a) shows a step motor used for running operating the precise $z$-axis cylinder in the feed room to provide powder and in the build room to perform sintering. Powder deposition thickness can be optimized by controlling the step motor. A roller was used to deposit powder (Fig. 2(b)) and an LM guide using the AC servo motor allowed the roller to perform accurate leveling movement (Fig. 2(c)). A roller rotated at a fixed speed to construct the horizontal powder surface. The horizontal transfer mechanism which controlled speed and torque consisted of two AC servo motors and a timing belt with speed reducer. The layer depositing cycle started by moving the powder up to the feeding room, followed by spreading a powder layer using a roller in the building room. The layer pattern was then scanned by the focusing the laser on the powder. Therefore, the position controlled stepping motor was very important to the process of lami-
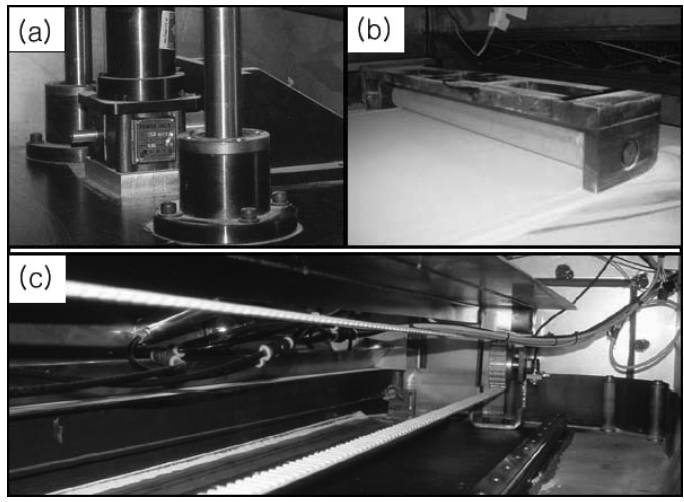

Fig. 2 Step motor, LM guide and roller mechanism

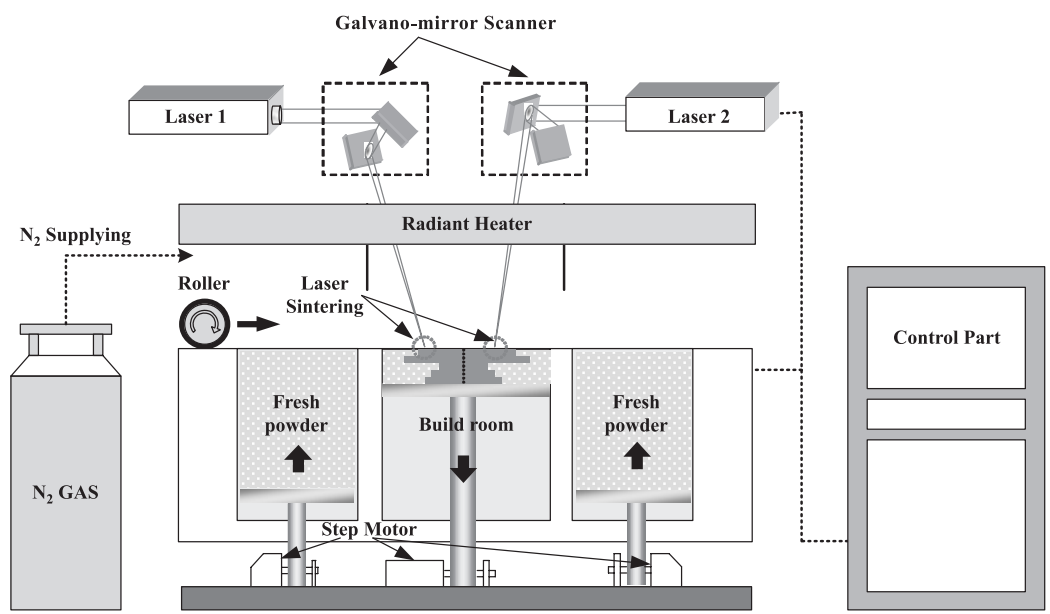

Fig. 1 Schematic diagram of SFF system 


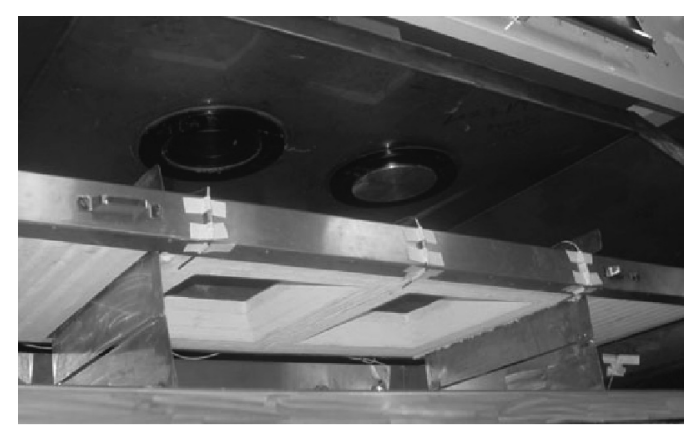

Fig. 3 Radiant heater part for SLS process

nating the powder. The roller mechanism had several specially designed features that influenced the roughness of the powder surface and the porosity of each powder, including the linear speed of the roller, the rotational speed of the roller, the feed ratio, the roller roughness, and the layer thickness.

\subsection{Heater module}

Laser sintering using polyamide powder in the SLS process required a build room temperature of $150^{\circ} \mathrm{C}$ or greater to preheat the powder. This study utilized a radiant heating system to preheat the polymer powder in the build room and the feed room. As shown in Fig. 3, the radiant heating system maintained an appropriate, uniform temperature in the build room and the feed room. Assuming that a constant laser power was used, if the temperature of the build room was too high, curling or over-melting of the prototype occurred. On the other hand, if the temperature of the build room was below the melting point temperature of the polymer, the prototype did not sinter properly and it could be demolished after sintering as a result of incomplete melting. The radiant heater system was controlled precisely by a PID control algorithm. The setting temperature of the heater was controlled within $1^{\circ} \mathrm{C}$. To minimize the difference between the surface temperature and the internal temperature of the powder, a plate-type piston heater and a cylindrical heater were installed in the build room (Fig. 8), in order to prevent curling that could occur during the sintering process.

\subsection{Dual laser module}

The optimum laser head unit was designed to implement the dual laser sintering method (Fig. 11). The laser was manufactured using a 3-axis, dynamic focusing lens such that a $500 \times 800 \times 500\left(\mathrm{~mm}^{3}, \mathrm{~W} \times \mathrm{D} \times \mathrm{H}\right)$ prototype could be fabricated using a dual laser. As shown in Figs. 4 and 5, the laser module consisted of a $\mathrm{CO}_{2}$ laser engine, beam expanders, reflection mirrors, a three-axis dynamic scanner, and an $x-y$ galvano mirror. The threeaxis dynamic scanner system was employed instead of an $\mathrm{f} \theta$ lens especially for sintering large objects. This scanner system had the ability to prevent spot distortion from occurring when the laser was used to irradiate large areas by applying a focal distance function. This three-axis dy-

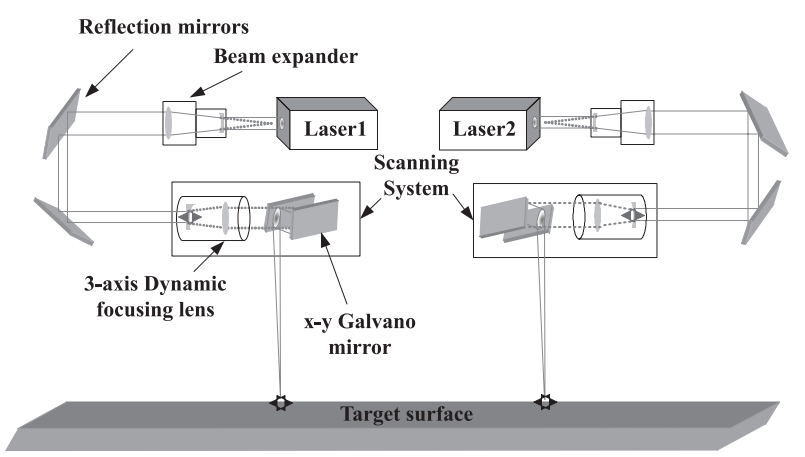

Fig. 4 Dual laser scanning system

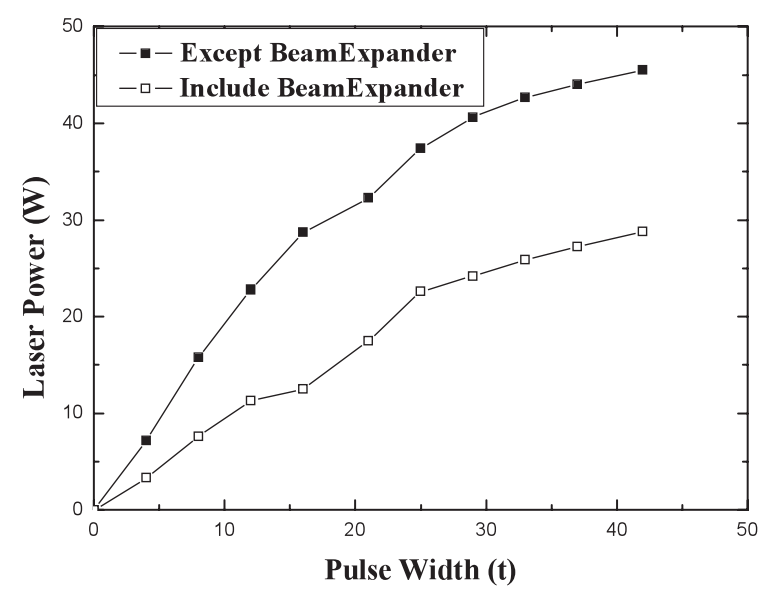

Fig. 5 Power curve of $\mathrm{CO}_{2}$ laser

namic scanner contained an objective lens and a concave lens located in front of the $x-y$ galvano mirror. Consequently, the laser system with dual lasers was able to sinter large-sized objects.

The laser beam diameter $\left(I / e^{2}\right)$ of the generated laser head was $1.8 \mathrm{~mm} \pm 0.2 \mathrm{~mm}$. Therefore, the diameter of the laser beam was enlarged acquire the desired spot size of $500 \mu \mathrm{m}$. The objective lens was fixed but the focal length was adjusted by moving the concave lens back and forth. When the laser beam was oscillated from the laser head to the beam expander, the amplitude of the laser beam was expanded as according to the magnification of the expander. Then laser beam from the beam expander become a parallel beam. The beam expander was located in front of the laser head in order that the laser beam from the laser head was disseminated more and more as the beam was operated. In other words, the dissemination of the laser beam was minimized by using the beam expander.

The beam expander used in this study consisted of a concave lens and an objective lens. The laser beam expanded when it passed through the concave lens but was collimated when it passed through the objective lens. The focal point of the concave lens and the objective lens were geometrically optically the same in order to produce expanded collimation. Figure 5 shows the power of the laser when it reached the surface of the powder to accomplish 
the sintering process. As shown in Fig. 12, when the pulse width was 42 us, the laser beam power decreased from 45 Watts to 29 Watts. Therefore, the loss of laser beam power was considerably high when the laser beam passed through this system.

\subsection{Nitrogen supplying module}

The nitrogen supply module was used to produce a nitrogen atmosphere in the work room and for the scanner lens. The nitrogen atmosphere controlled soot from micro explosions that can occur during laser sintering within the system and prevented the powder from blowing and sticking to the scanner lens. A nitrogen atmosphere, $>95 \%$, should exist during laser sintering. An oxygen sensor capable of detecting less than 5\% oxygen was used to determine if the nitrogen atmosphere was created properly.

\section{Scan Path Generation}

Scan paths consist of two parts. One is laser marking from the sliced section, and the other is jumping to next scan path. To generate these scan paths, first of all, scan spacing, the diameter of laser beam, and scan speed have to be considered. Scan paths are generated from these parameters, and scan control is needed to follow generated scan paths precisely and to enable the fabricated surface to absorb fine energy in order to enhance dimensional accuracy. Scan path generation algorithm affects the accuracy and total time of manufacturing. On the characteristics of SLS machine using sintering powder by thermal energy, there is a time delay between the sintered and sintering surface. It results in shrinkage, curling and warpage by thermal distribution. The reduction of thermal distribution can lead to the better precision and quality of the fabricated part. To do this, it is important to scan the surface of powder fast. The shorter the fabricating time in one layer is, the lesser the thermal distribution will be, resulting in a higher quality.

\subsection{Scan path generation}

From sliced sections, scan points can be calculated by obtaining intersection points in one layer as shown in Fig. 6. In advance, the starting and end points of the layer have to be calculated to scan each layer. Scanning ray moves as scan spacing along perpendicular of the scanning direction. Unnecessary calculation could be removed by calculating only scan points when there are intersection points between each loop and moving scanning ray. After intersection points between scanning ray and the line of each loop are found, final scan points will be determined by sorting obtained raw scan points. Laser beam moves to first scan point with 'Laser Off', and to next scan point with 'Laser On'. Commands "mark" and "jump" complete the toggle of laser operation according to the order.

\subsection{Eliminating error points}

If scanning spots are adopted by estimating intersection points with contour line moving as wide as scan

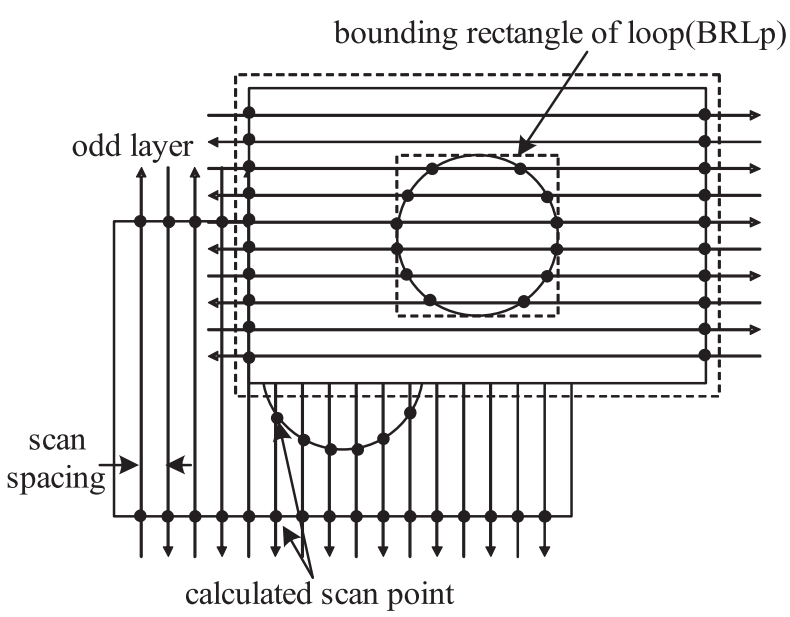

Fig. 6 Calculated scan points

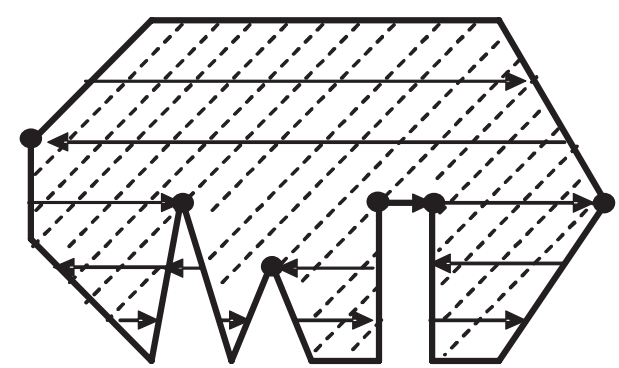

- : Calculated scan points intersected with contour point

$\vdash$ : Scan path (from laser 'on' to 'off')

せ: Hatching region

Fig. 7 Special case for intersection with contour points

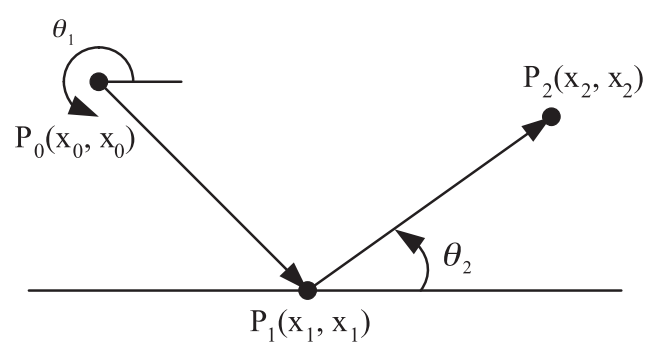

Fig. 8 Vector representation of neighborhood segment

space, error can be occurred as shown in Fig. 7. Scanning path should have even-numbered pairs consisting of starting point and ending point. However, if a contour point, not contour line segment, on closed curve is estimated as a scanning spot, odd-numbered scanning spots are generated. This leads to a serious error in scanning path as shown in Fig. 7. Therefore, selecting contour point as scanning spot, the adopted points and discarded points should be chosen. In order to represent this case in general, neighboring contour lines are indicated as vector as shown in Fig. 8. $\theta_{1}$ and $\theta_{2}$ indicate counter-clockwise angle from $X$-axis as in the expressions equations (1) and (2). 


$$
\begin{aligned}
& \theta_{1}=\cos ^{-1}\left(\frac{x_{1}-x_{0}}{\sqrt{\left(x_{1}-x_{0}\right)^{2}+\left(y_{1}-y_{0}\right)^{2}}}\right) \\
& \theta_{2}=\cos ^{-1}\left(\frac{x_{2}-x_{1}}{\sqrt{\left(x_{2}-x_{1}\right)^{2}+\left(y_{2}-y_{1}\right)^{2}}}\right) \\
& 0^{\circ}<\theta_{1} \leq 180^{\circ} \text { and } 0^{\circ} \leq \theta_{2}<180^{\circ} \\
& 180^{\circ}<\theta_{1} \leq 360^{\circ} \text { and } 180^{\circ} \leq \theta_{2}<360^{\circ}
\end{aligned}
$$

When $\theta_{1}$ and $\theta_{2}$ satisfy the conditions of the Eq. (3) simultaneously according to each vector evaluated from the Eqs. (1) and (2), a contour point must be selected as a scanning spot.

\section{Sintering Parameters Experiments}

The $\mathrm{CO}_{2}$ laser emitted infrared radiation of the wavelength $10.6 \mu \mathrm{m}$. Most plastics are transparent in this near-infrared wavelength region which corresponds to $8 \sim 10 \mathrm{~kJ} / \mathrm{mole}$. This level of energy was adequate to sinter the polymer powder although it was different from the laser beam power. The polymer used in the laser sintering process was composed primarily of combined $\mathrm{C}-\mathrm{C}$ or $\mathrm{C}-\mathrm{O}$ bonds and melted due to the rapid increase in vibration when the wavelength of the irradiated beam was about $10 \mu \mathrm{m}^{(7)}$. The polyamide powder was sintered through this process and the data scanned by CAD files for the SLS process were converted into the STL-file format and sliced in $100 \mu \mathrm{m}$ units against the $z$ axis. The starting material used for the sintering parameter experiment was polyamide powder having the characteristics presented in Table 1. Figure 9 illustrates the block diagram of the SLS process used to manufacture a product. A nitrogen atmosphere $(>95 \%)$ was first created through nitrogen injection. The temperature on the surface of the powder surface was controlled in the build room and in the feed room. Powder deposition and $\mathrm{CO}_{2}$ laser beam irradiation were repeated alternately to fabricate a multi-layer, solid

Table 1 Specification of polyamide power

\begin{tabular}{|c|c|c|c|}
\hline & Density $\left(\mathrm{g} / \mathrm{cm}^{3}\right)$ & $\begin{array}{c}\text { Particle size } \\
\text { average }(\mu \mathrm{m})\end{array}$ & Melting point $\left({ }^{\circ} \mathrm{C}\right)$ \\
\hline $\begin{array}{c}\text { Polyamide } \\
\text { powder }\end{array}$ & 0.59 & 58 & 184 \\
\hline
\end{tabular}

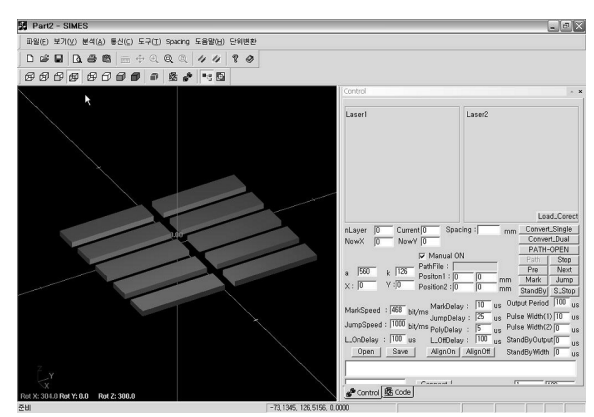

freeform. Sintering variable determination tests were performed repeatedly on samples of constant shape and work room temperature, $\mathrm{CO}_{2}$ laser scan area, scanning speed, and laser beam power (Table 2). By controlling the on/off delay, mark delay, and jump delay of the laser, microcurling was prevented from occurring at the beginning and ending points of the laser scanning path. Figure 10 shows samples fabricated using the sintering parameters experiment with a CAD model.

\section{Solid Freeform Fabrication Experiment}

\subsection{SFF experiment with single laser}

By applying fabricating parameters obtained from the sintering parameters experiments using a single laser, the first pyramid-shape model was fabricated from CAD data (Fig. 11). To reduce the curling rate for the large area and increase bonding strength between layers a laser scanning

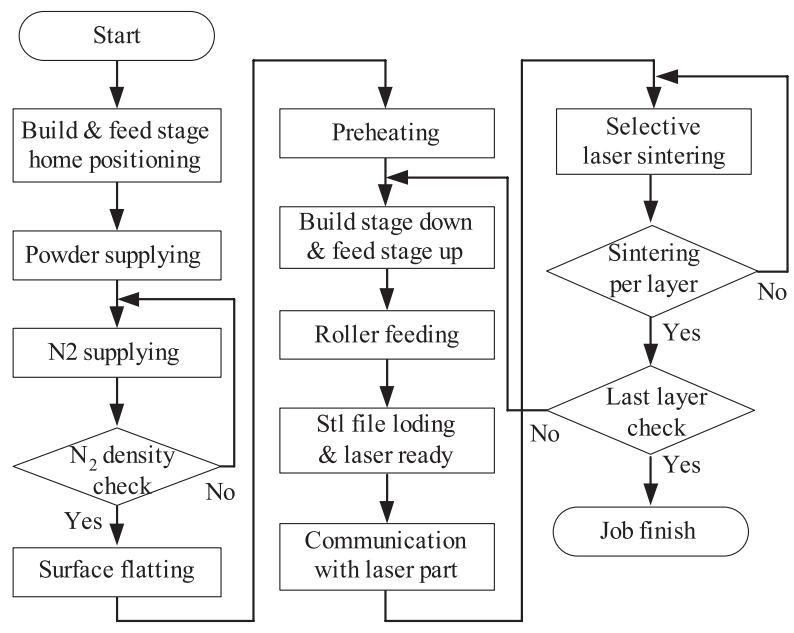

Fig. 9 Block diagram of SLS process

Table 2 Test of sintering process variables

\begin{tabular}{|c|c|c|}
\hline & Test & Results \\
\hline Temperature $\left({ }^{\circ} \mathrm{C}\right)$ & $150-165$ & $155 \sim 160$ \\
\hline Scan speed $(\mathrm{m} / \mathrm{s})$ & $1-10$ & $3.5 \sim 7$ \\
\hline Scan space $(\mathrm{mm})$ & $0.1-0.5$ & $0.3 \sim 0.5$ \\
\hline Laser power $($ Watts) & $10-20$ & $12 \sim 18$ \\
\hline Layer thickness $(\mathrm{mm})$ & $100 \sim 150$ & 100 \\
\hline
\end{tabular}

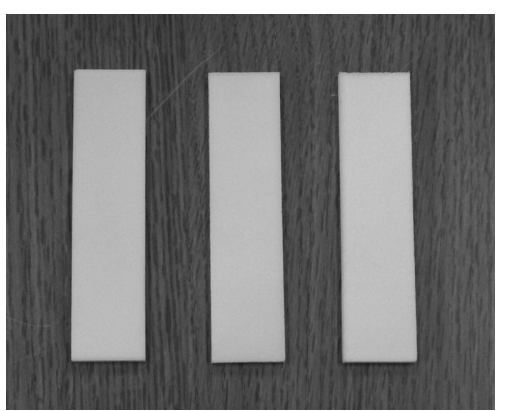

Fig. 10 Fabricated specimens from variable test 

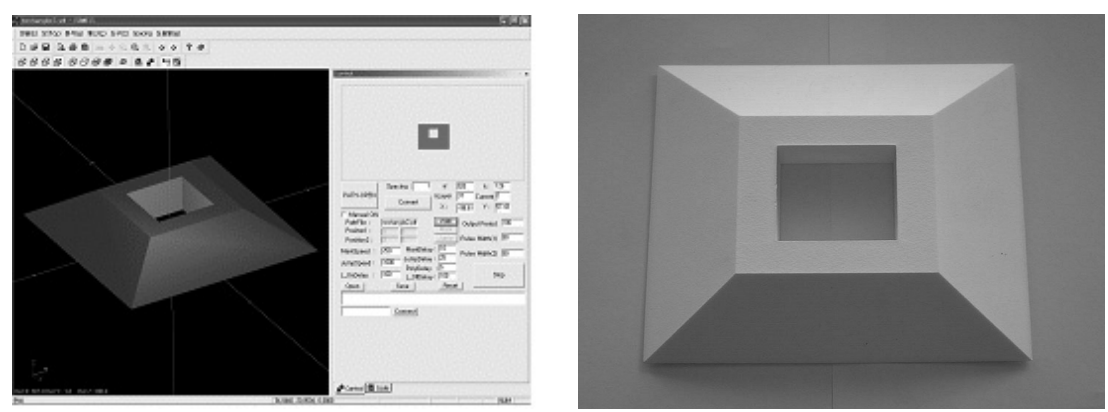

Fig. 11 Fabricated 3D object using the STL file
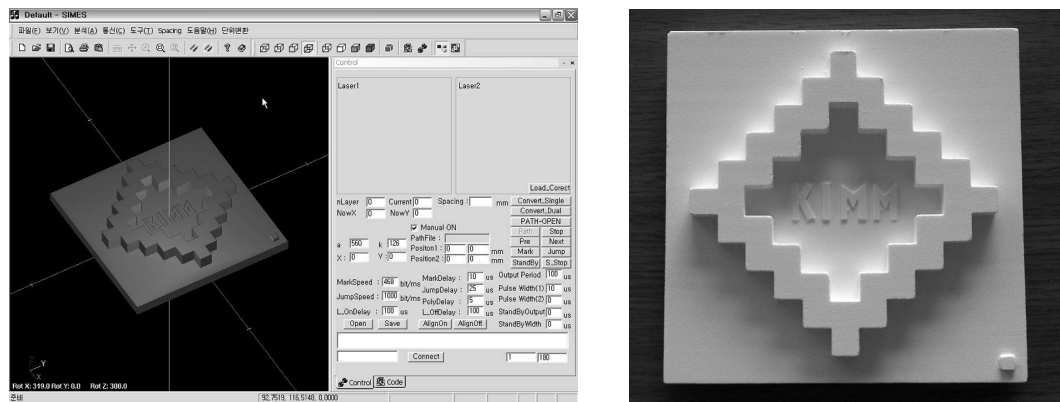

Fig. 12 Fabricated 2nd 3D object using the STL file

Table 3 Sintering conditions of samples

\begin{tabular}{|c|c|c|}
\hline & $1^{\text {st }}$ Sample & $2^{\text {nd }}$ Sample \\
\hline Temperature $\left({ }^{\circ} \mathrm{C}\right)$ & 155 & 155 \\
\hline Scan speed $(\mathrm{m} / \mathrm{s})$ & 5 & 6 \\
\hline Scan space $(\mathrm{mm})$ & 0.3 & 0.4 \\
\hline Laser power $(\mathrm{Watts})$ & 15.4 & 17.2 \\
\hline Layer thickness $(\mathrm{mm})$ & 100 & 100 \\
\hline
\end{tabular}

path was generated for each layer. Consequently, the curling rate decreased by approximately $25 \%$ and the bonding strength between layers increased, as compared with laser scanning in the same direction. To consider sintering parameters for more complicated models, a second model were fabricated (Fig. 12). Table 3 presents the sintering conditions used for generating the first and second models. According to Fig. 13, Table 4 presents the results of dimension errors between a 3D CAD model and a fabricated 2nd sample. The experimental results demonstrated that the dimension error rate was less than $1 \mathrm{~mm}$ for each measurement point.

\subsection{SFF experiments with dual lasers}

When fabricating a solid freeform using a dual laser, both the sintering level and mechanical properties depended on the overlap rate of the two laser beams, the accuracy of $x$ and $y$ coordinates, and the layer scanning path generation methods. Figure 14 illustrates the overlapped section of the sample selected for a sintering process experiment using a dual laser. The sintering experiment was conducted under the conditions specified in Table 5. Five samples were fabricated using values of 0,3 ,

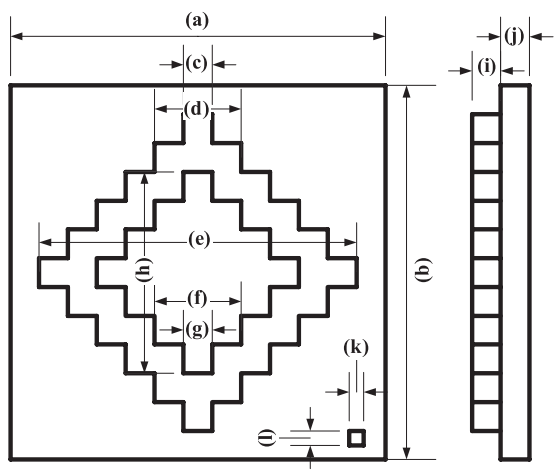

Fig. 13 Measurement point fabricated 2nd 3D object

Table 4 Results of dimension error between the 3D CAD model and fabricated 2 nd object

\begin{tabular}{|c|c|c|c|c|c|c|}
\hline & (a) & (b) & (c) & (d) & (e) & (f) \\
\hline 3D model (mm) & 160 & 160 & 12 & 35 & 135 & 40 \\
\hline Fabricated sample (mm) & 159.62 & 159.67 & 11.31 & 34.18 & 134.01 & 39.2 \\
\hline Error (mm) & 0.38 & 0.33 & 0.69 & 0.82 & 0.99 & 0.8 \\
\hline & (g) & (h) & (i) & (j) & (k) & (l) \\
\hline 3D model (mm) & 12 & 90 & 10 & 12 & 6 & 6 \\
\hline Fabricated sample (mm) & 11.96 & 89.9 & 9.72 & 1185. & 5.88 & 5.91 \\
\hline Error (mm) & 0.04 & 0.1 & 0.28 & 0.15 & 0.12 & 0.09 \\
\hline
\end{tabular}

6, 9 and $12 \mathrm{~mm}$, respectively, for the overlap rate based on the center of each sample. Figure 15 shows a sample fabricated using the sintering process with a dual laser, which has lower dimensional accuracy at the overlapped section than the product fabricated by a single laser.

\section{Conclusions}

This paper presented development of component 

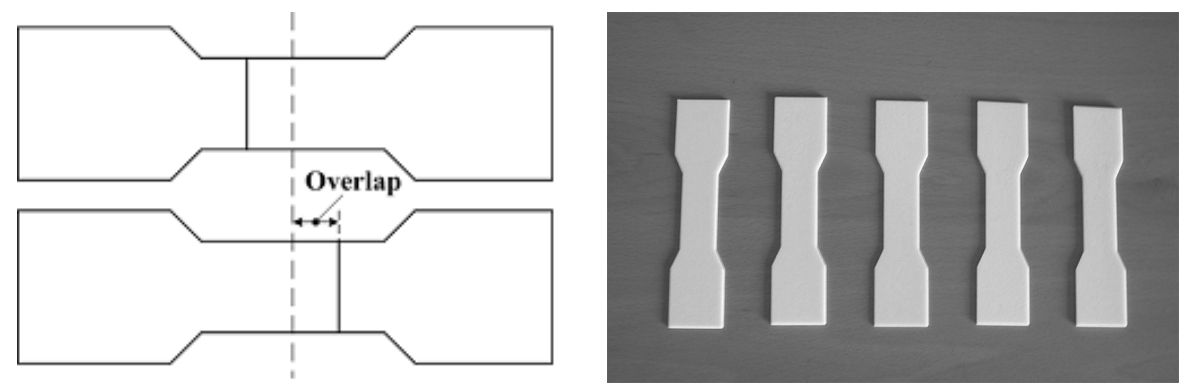

Fig. 14 Scan path generation and fabricated samples for dual laser sintering
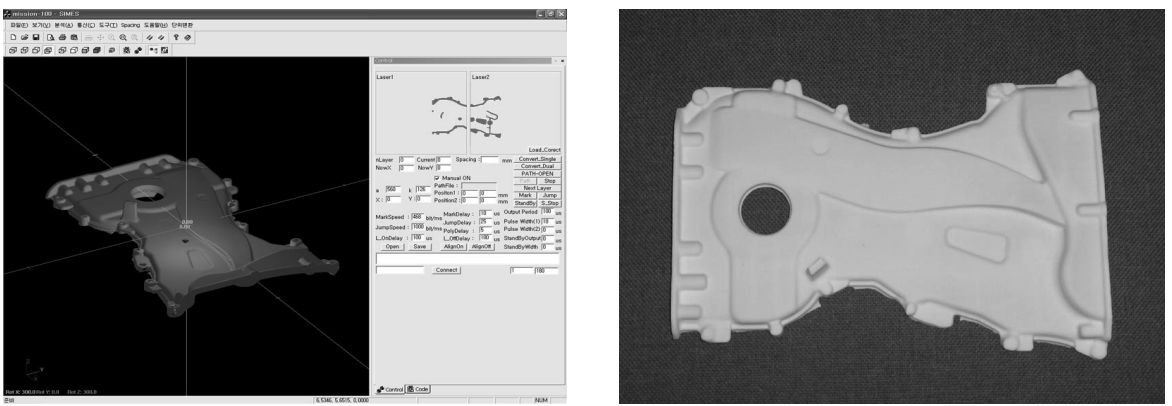

Fig. 15 Fabricate sample by dual laser sintering

Table 5 Sintering conditions of dual laser sample

\begin{tabular}{|c|c|}
\hline & Sintering conditions \\
\hline Temperature $\left({ }^{\circ} \mathrm{C}\right)$ & 165 \\
\hline Scan speed $(\mathrm{m} / \mathrm{s})$ & 4.5 \\
\hline Scan space $(\mathrm{mm})$ & 0.4 \\
\hline Laser power $($ Watts $)$ & 16 \\
\hline Layer thickness $(\mathrm{mm})$ & 100 \\
\hline Overlap rate $(\mathrm{mm})$ & $0,3,6,9,12$ \\
\hline
\end{tabular}

technologies for an SFF system with the dual laser system. In addition, the sintering parameters were obtained for single and dual laser systems by investigating sintering experiments for a polyamide starting material. The sintering properties of each system were considered. The results obtained from experiments designed to test the SFF system developed included: 1) Through analysis and experimentation on each module of the SFF system developed, component technologies were developed and a solid freeform was fabricated from CAD files; 2) a scanning path was generated and used to fabricate a solid freeform from STL data; and, 3) a dual laser system that enabled large-area fabrication was constructed and a sintering process and sintering properties were considered.

\section{Acknowledgments}

This study was sponsored by "Three-Dimensional Digital Real Object Duplication System (RODS)” Development Project from the Ministry of Commerce, Industry and Energy. We appreciate and thank everyone who has supported and participated in this study.

\section{References}

( 1 ) Andreas, G., Rapid Prototyping, 1st Edition, (2003), pp.29-30.

( 2 ) Ashley, S., Rapid Prototyping Systems, Mechanical Engineering, (1991), pp.34-43.

( 3 ) Agarwala, M., Bourell, D.L., Wu, B. and Beaman, J.J., An Evaluation of the Mechanical Behaviour of BronzeNi Composite Produced by Selective Laser Sintering, Proceedings of the Solid Freeform Fabrication Symposium, University of Texas at Austin, USA, (1993), pp.193-203.

( 4 ) Kuczynski, G.C. and Zaplatynskyj, I., Sintering of Glass, J. Am. Ceram. Soc., Vol.39, No.10 (1956), pp.349-350.

( 5 ) Chen, T. and Zhang, Y., A Partial Shrinkage Model for Selective Laser Sintering of a Two-Component Metal Powder Layer, International Journal of Heat and Mass Transfer, Vol.49, Issues 7-8 (2006), pp.1489-1492.

( 6 ) Forderhase, P., McAlea, K., Michalewicz, M., Ganninger, M. and Firestone, K., SLS ${ }^{\mathrm{TM}}$ Prototypes from Nylon, Proceedings of Solid Freeform Fabrication Symposium, (1994), pp.102-109.

( 7 ) Chen, K., Crawford, R.H. and Beaman, J.J., Parametric Representation of Part Contours in SLS Process, Presented at 1996 Solid Freeform Fabrication Symposium, Austin, TX, August 12 (1996), pp.597-608.

( 8 ) Chua, C.K., Leong, K.F. and Lim, C.S., Rapid Prototyping: Principles and Applications, 2nd Edition, (2003), pp.10-12, World Scientific Publishing.

( 9 ) Dutta, D., Improving Surface Quality and Throughput in Layered Manufacturing by Adaptive Slicing, The Seventh International Conference on Rapid Prototyping, (1996), pp.94-101.

(10) Barlow, J.W., Sun, M.M. and Beaman, J.J., Analysis 
of Selective Laser Sintering, In Conference Proceedings, Second International Conference on Rapid Prototyping, Lightman, A.J. and Chartoff, R.P., University of Dyaton: Dyaton, Ohio, (1991), pp.1-14.

(11) Park, S.-M., Crawford, R.H. and Beaman, J.J., Sliced Geometry Representation Based on Implicit Curves, Presented at the 2000 Computers and Information in Engineering Conference, Baltimore, MD, September 12, (2000), pp.105-112.

(12) Yang, J., Bin, M., Zhang, X. and Liu, Z., Fractal Scanning Path Generation and Control System for Selective Laser Sintering(SLS), International Journal of Machine
Tools \& Manufacture, Vol.34 (2003), pp.293-300.

(13) Yang, Y., Loh, H.T., Fuh, J.H. and Wang, Y.G., Equidistant Path Generation for Improving Scanning Efficiency in Layered Manufacturing, Rapid Prototyping Journal, Vol.8, No.1 (2002), pp.30-37.

(14) John, D.W. and Carl, R.D., Advances in Modeling the Effects of Selected Parameters on the SLS Process, Rapid Prototyping Journal, Vol.4 (1998), pp.90-100.

(15) Benda, J., Temperature Controlled Selective Laser Sintering, Proceeding of the Solid Freeform Fabrication Symposium, University of Texas at Austin, TX, Vol.5 (1994), pp.277-284. 